\title{
Kein Ende in Sicht. Zur Rolle der Ideologie in der Politik - aus Sicht der Politischen Theorie
}

\section{Ideologische Theorie der Ideologie}

Am Ende triumphiert die Vernunft in Form der liberalen Demokratie. Diese populäre Deutung, vorgelegt von Francis Fukuyama (1992), hat mittlerweile an Überzeugungskraft eingebüßt. Die Sicherheit, an der Schwelle einer „New Global Order“ (George Bush Sen.) zu stehen, in der nach dem Untergang der Sowjetunion die (liberale) Freiheit in Form von Menschenrechten und demokratischer Selbstbestimmung zum Allgemeingut wird, ist gewichen. Geblieben ist allenfalls das Unverständnis, warum sich die vermeintlich überlegene, nicht auch als alternativlose Ordnungsform in den verschiedenen Regionen der Welt durchsetzt. Führt womöglich nicht die Politik, sondern die Ökonomie ans Ende der Geschichte? Genauer betrachtet stand hinter Fukuyamas Posthistoire-These weniger Hegel, sondern mehr Kojève (2005) und dessen einflussreiche, marxistisch inspirierte Lesart der Hegelischen Phänomenologie des Geistes: Die „Aufhebung“ der Anerkennungskämpfe läutet die letzte Runde der Weltgeschichte ein, wobei Alexandre Kojève dem Kapitalismus - anders als Marx - nicht nur die Fähigkeit zusprach, als Produzent von Wohlstand aufzutreten, sondern auch als dessen Verteiler. Die Folge davon: Die sozialen Härten des Marktes bleiben aus, und ebenso die des Klassenkampfes. Die Befreiung von allem Bedrängenden realisiert sich ohne Revolution; und doch offenbart sich diese Entwicklung auch als ein Verfallsprozess: Mit erfolgreicher Emanzipation degeneriere zugleich die „Negativität" des Menschen und damit sein Wille, sich selbst zu überwinden, seine Fähigkeit, die eigene Natur transzendieren zu können, so Kojève in einer unverkennbaren nietzscheanischen Wendung. Nietzsche (1955: 284) selbst hatte seinerseits im Vorgriff auf das Ende der Geschichte den „letzten Menschen“ als korrumpierte Existenz beschrieben, der nur noch „blinzelnd“ sein „Lüstchen“ vor Augen hat.

Solche Endzeitimaginationen, auch in ihrer ambivalenten Version, sind Großaufnahmen einer ideologiefreien Welt. Posthistoire setzt Postideologie voraus. Der Kampf der Ideen schwächt sich ab, Ruhe tritt ein - und mag es auch die „Ruhe des Friedhofs" (Don Carlos) sein. In einem etwas kleineren Format findet sich eine solche Position in der „End of Ideology“-These wieder, die in prominenter Form vor allem von Raymond Aron (1955), Daniel Bell (1962) und Edward Shils (1955) 
vertreten worden ist. Hier sind es nicht die Kopien des Weltgeistes oder die tiefere Einsicht in den Gang der Geschichte, sondern empirische Beobachtungen, mit der die These gestützt werden soll; so etwa die Beobachtung einer Systemkonvergenz zwischen Ost und West beim Ausbau des modernen Wohlfahrtsstaates oder die abnehmende Trennkraft politischer Parteien im Links-Rechts-Kontinuum (Lipset 1990: 101 ff.). Im Lichte der vorangegangenen ideologischen Gegensätze und deren Zugkraft für die Mobilisierung der Massen in den 1930er und 1940er Jahren erschien es plausibel, von einer Zäsur gegenüber der ,Zeit der Ideologien“ (Bracher 1982) auszugehen. Bis die Kombination aus Bürgerrechtsbewegung, Entkolonialisierung, Vietnamkriegsgegnerschaft und Achtundsechziger-Protest die Gesellschaften ziemlich durcheinander wirbelte; ein gefundenes Fressen für alle Kritiker der „End of Ideology"-These (vgl. Lloyd 2003: 220-222). Nur ergibt sich auch aus dieser kritischen Warte kein Beitrag für einen politiktheoretischen Zugang zum Phänomen der Ideologie, solange diese an den historischen oder gesellschaftlichen Umbrüchen kleben bleibt. Denn Ideologie beweist ihre Relevanz nicht nur - und nicht einmal vorrangig - in ihrer Kraft zu spalten, sondern mindestens ebenso in ihrer Macht zu vereinen. Es sind nicht nur die Konflikte, die Brüche und Umbrüche, die lauten Leidenschaften, in denen sie sich als Herrin der Lage erweist; ihr Metier ist ebenso Konsens, Fortbestand und Rationalisierung. Macht man sich die (ihrerseits umstrittene) Postdemokratie-These zu eigen (Crouch 2004; Wolin 1996) - wie in prominenter Weise von konservativer Seite durch den kürzlich verstorbenen Publizisten und FAZ-Herausgeber Frank Schirrmacher (2011) -, so lässt sich aus dieser Perspektive die Subversion demokratischer Willensbildungs- und Entscheidungsprozesse durch neo-liberale Interessen rekonstruieren. Postdemokratie ist mithin das Gegenteil von Postideologie, vielmehr besteht das ideologische Moment, so die These, im parasitären Einnisten in die Strukturelemente moderner Massengesellschaften und deren Verfahren zur Selbstbeschreibung, in denen sich der Glaube an Demokratie, Verfassungsstaatlichkeit und sozialer Marktwirtschaft widerspiegelt. Der Ertrag der Postdemokratie-These kann hier dahinstehen (Ritzi 2014); sie erweitert jedoch zumindest den Horizont für die Funktionsfähigkeit und Flexibilität ideologischer Mechanismen - und legt folgende Einsicht nahe: Um die Frage nach der gegenwärtigen Rolle der Ideologie zu beantworten, muss man anders ansetzen als bei den vermeintlichen Konjunkturen. Eine Konjunkturtheorie ist eine ideologische Theorie der Ideologie. 


\section{Kritik der Kritik der Aufklärung}

Als im Nachklang der Französischen Revolution Antoine Destutt de Tracy den Begriff der Ideologie erstmalig ins Spiel brachte, war dies mit dem Vorhaben verbunden, eine systematische Analyse und Bewertung von Gedanken und Handlungsweisen vorzunehmen, um schrittweise zu einer Wissenschaft der Ideen vorzudringen, aus der sich Gesetzmäßigkeiten und Verbesserungsmöglichkeiten ableiten lassen (Kennedy 1978). Ein ebenso optimistisches wie positivistisches Unterfangen. Der Begriff selbst erwies sich als „Non-Starter“: Ideologie wurde - unter anderem von Napoleon und Hegel - zum Inbegriff für leeres Gerede über Politik. Der Sache nach jedoch war ein Großteil der Aufklärung eine frühe Form von Ideologiekritik. Vor allem die Riege der Junghegelianer - darunter solche Denker wie Bauer, Feuerbach und Stirner - drang auf eine radikale Form der Kritik an den bestehenden Verhältnissen und deren korrumpierende Wirkung auf Wahrheitsansprüche. Ihr Credo: Die Philosophie kennt neben der (haltlosen) Meinung noch einen zweiten Feind, nämlich die Machtstrukturen, auf denen die jeweilige Ordnung beruht. Platons Strategie, durch Erkenntnistheorie dem Verwirrspiel der Sophisten entgegenzutreten und deren Lehren als bloße Meinung (doxa) zu entlarven, ist durch Herrschaftskritik zu ergänzen, die sich gegen das System der Indoktrinierung durch Kirche und Staat zu richten hat, um den Eigennutz dieser Gemeinwohlansprüche offenzulegen.

Von Beginn an, so lässt sich abgekürzt sagen, wohnt dem Ideologiebegriff eine deskriptive und eine pejorative Dimension inne: Jene zielt im Sinne de Tracys auf die Beschreibung und Systematisierung der Elemente von Ideensystemen; diese macht die interessengeleitete Kopplung von Macht (Herrschaft) und Wissen (Wahrheit) zum Gegenstand der Kritik. Beide Ursprünge sind nach wie vor für das Verständnis relevant. Seinen eigentlichen Theorieschliff hat der Begriff jedoch erst in der Auseinandersetzung mit der Ideologiekritik von Marx und Engels erhalten.

In der Schrift über Die deutsche Ideologie (1845/46) wird ein radikaler Ansatz vorgelegt: eine Kritik der Ideologiekritik, die den Junghegelianern im Kern vorhält, naiv zu argumentieren und dadurch selbst Teil jener Ideologie zu sein, die sie zu kritisieren beabsichtigen. Der Vorwurf der Naivität bezieht sich auf ,[d]iese unschuldigen und kindlichen Phantasien“, wie Marx und Engels höhnen (MEW 3: 13), denen zufolge man durch eine Kritik des Denkens ein Herrschaftssystem ins Wanken bringen könne, dessen Stabilität doch aber gerade daraus resultiert, dass es über seine eigenen Reproduktionsbedingungen herrscht. Die Bewusstseinsänderung geht eben nicht den materiellen Veränderungen voraus, sondern ist eine Folge von ihnen (26). Eine Ideologiekritik, die sich selbst über diesen Zusammenhang von Ursache 
und Wirkung täuscht, kann keine Massen über deren Selbsttäuschung aufklären, sondern letztlich nur dazu beitragen - zur Täuschung, versteht sich, nicht zur Aufklärung (20). Anders gewendet: Naive Aufklärer sind keine; sie sind Knechte im Dienste des Systems. Man könnte hinzufügen: unfreiwillige Knechte - und dieser Zusatz führt zu den tieferen Schichten der komplexen Argumentation von Marx und Engels: Denn eine wesentliche Pointe des bekannten Bildes von der Camera obscura besteht ja darin, dass die „verkehrte Welt" der einzelnen Person suggeriert, dass diese macht, was sie denkt. Ein „objektiv falsches Bewusstsein“, denn tatsächlich macht der Einzelne das, was andere denken, was gemacht werden soll. Der ideologische Prozess verkehrt das Primat zwischen Sein und Bewusstsein mit der Folge, dass der Einzelne seine heteronome Ausbeutung für autonome Selbstbestimmung hält - und sich infolge dieser Selbsttäuschung von sich selbst entfremdet. Ideologie ist hiernach keineswegs ,nur“ eine Störung der Wahrnehmung; sie ist ihrer Struktur nach perfide, weil sie die Mündigkeit hintertreibt - und dadurch zu einem idealen Machtinstrument der herrschenden Klasse wird.

Die deutsche Ideologie war ein Text zur „Selbstverständigung“ zwischen Marx und Engels und ist im Grunde über dieses Entwurfsstadium nicht hinausgelangt (Bohlender 2010: 42). Aber er hat den Ideologiediskurs nachhaltig beeinflusst, wozu zumindest zwei Gründe maßgeblich beigetragen haben: Erstens verweist der Text auf die Selbstreflexivität der Ideologie als Struktur, die ihre eigenen Produktionsbedingungen zum Gegenstand hat; zweitens sensibilisiert er für die Bedeutung der Kontextualisierung ideativer Elemente in institutionellen Settings, um die Verbindung von Wissen und Macht zu invisibilisieren. Eine der größten Schwächen der Konzeption von Marx und Engels besteht jedoch darin, Ideologie als einheitliches und zugleich ephemeres Phänomen zu behandeln. Der Kapitalismus ist eine Ideologie, aber nicht jede Ideologie heißt Kapitalismus - und gräbt zusammen mit diesem ihr eigenes Grab.

\section{Produktivität, Pluralität, Kontinuität}

Wie lassen sich Pluralität und Kontinuität der Ideologie denken, ohne die zentralen Einsichten von Marx und Engels aufzugeben? Für die aktuelle Ideologieforschung haben sich drei Ansätze als wegweisend erwiesen. Zunächst sind die Arbeiten von Karl Mannheim zu nennen, darunter insbesondere Ideologie und Utopie (1995 [1929]), in denen er vor allem die Ubiquität der Ideologie als Phänomen der sozialen Welt erfasst und begründet. Mannheim teilt Marxens Auffassung, dass Ideologie die sozialen Umweltbedingungen und insoweit auch die strukturellen Machtverhältnisse reflektiert. Anders als Marx sieht er jedoch in der Ideologie keine exklusive 
Eigenschaft der kapitalistischen Klassengesellschaft; vielmehr haben Marx und Engels nur ein Beispiel für einen „totalen Ideologiebegriff“ geliefert, indem sie die Täuschung und Selbsttäuschung als systemimmanente Entsprechung der Klassendifferenz dargelegt haben (1995: 55, FN 3). Darüber hinaus, so Mannheim, existiert auch ein ,partikulärer Ideologiebegriff“ (1995: 53 ff.), der sich auf die psychologische Dimension (Motive, Intentionen etc.) beschränkt und im Sinne des Ideologievorwurfs sich auf bestimmte Inhalte des Gegners bezieht. Auf Grundlage dieser Differenzierung lässt sich die Allgegenwärtigkeit der Ideologie erfassen und vermessen. Sie ist Teil gesellschaftlicher Lebenszusammenhänge, ebenso unvermeidlich wie veränderlich: Weil innerhalb der Totalität der Sozialität jede Sichtweise notwendig eine Perspektive darstelle, ist letztlich alles Denken - auch das eigene stets ideologisch (1995: $102 \mathrm{ff}$.), und zwar bereits wegen seiner Partikularität sowie überdies wegen des Grundzugs zur Verabsolutierung (und damit zur realitätsverzerrenden Darstellung) der partikulären Position. Hinzutritt die von Marx und Engels offenbarte strukturelle Bedeutung der sozioökonomischen Umweltbedingungen, die sich gleichwohl verändern können. Mannheim hat den Anspruch erhoben, seine epistemische Position des „dynamischen Relationismus“"vom (ethischen) Relativismus unterscheiden zu können (1995: 71 ff., 258, 262) - und dabei seine Hoffnungen auf die ,freischwebende Intelligenz" gesetzt (1995: 134-143), die sich (contra Marx) von ihren konkreten soziökonomischen Lebenslagen lösen und als Produzenten vernünftigen Wissens auftreten könne.

Mit Mannheims Weichenstellungen wird der Ideologiebegriff maßgeblich ausgeweitet und aufgewertet - in einer Weise, die an das Anliegen von Destutt de Tracy erinnert. Drei wesentliche Einsichten lassen sich aus seinen Analysen gewinnen: Erstens erweitert sich das Funktionsspektrum der Ideologie, die sich nicht mehr in der manipulativen Steuerung der Herrschaftsunterworfenen erschöpft; zweitens löst sich die Ideologie vom Kapitalismus ab und tritt dadurch fortan im Plural auf; und drittens verliert Ideologie ihren ephemeren Status. Soziale Gruppen mögen kommen und gehen, immer jedoch gehört zu ihrer Bildung und Beständigkeit ein ideologisches Element hinzu. Das Ende der Klassengesellschaft wäre für Mannheim eben nicht (wie für Marx) gleichbedeutend mit dem Beginn eines postideologischen Zeitalters.

Für die Rezeption der Ideologiekonzeption von Marx und Engels innerhalb der marxistischen Tradition sind zwei Denker hervorzuheben: Antonio Gramsci und Louis Althusser. Sie haben beide gleichzeitig und maßgeblich über ihre jeweilige Ideologiekonzeption das post-marxistische Denken befördert: Gramsci hat den Ideologiediskurs vor allem um zwei Perspektiven bereichert, die beide in einer gewissen geistigen Verwandtschaft zu Mannheim stehen, nämlich die Bedeutung der 
Zivilgesellschaft als Medium des ideologischen Denkens und die relative Autonomie der politischen Eliten; beide Elemente hängen mit Gramscis Hegemoniebegriff zusammen. Zur Rekonstruktion dieses Ansatzes kann Marxens bekanntes Diktum dienen, wonach ,[d]ie Gedanken der herrschenden Klasse [...] in jeder Epoche die herrschenden Gedanken" seien (MEW 3: 46). Gramsci füllt diesen Satz mit Leben, indem er die Eigenart der Ideen von jener der Gewalt abhebt. Sinn und Wirkung von Ideen zielen hiernach gerade nicht auf die Gewaltanwendung oder Zwangsandrohung, sondern auf die Möglichkeit der Führung durch Zustimmung. Folgerichtig wird zwischen Herrschaft und Führung unterschieden (1998 [1934/35]: 1947 [§24]): Während Herrschaft sich im Zweifel auf das Gewaltmonopol des Staates zurückziehen kann, stützt sich politische Führung auf eine gesellschaftlich dominante Position - von Gramsci „Hegemonie“ genannt -, die aus dem ideologischen Ringen um Zustimmung hervorgeht. Als Medium, in dem die Lager für ihre Positionen werben und die Vorhaben entsprechend „framen“ können, besitzt Ideologie eine handlungstheoretische Dimension. In Gramscis Metaphorik handelt es sich um einen Stellungskrieg, in dem um den Ausbau von Positionen (in der Regel mühsam) gerungen wird - und der dem Sieger infolge des politischen Geschicks und des Organisationsvermögens die Möglichkeit bietet, die Früchte hegemonialer Gestaltungsfreiheit zu ziehen (Barret 1991: 54). Bei Gramsci liegt der Akzent auf der Konkurrenz, bei Mannheim eher auf der Objektivität - beide konzipieren jedoch die Rolle gesellschaftlicher Eliten als aktive ideologische Produzenten mit der Fähigkeit, im Rahmen gesellschaftlicher Konfliktlagen Konsensorientierungen herzustellen - eine Idee, die politikwissenschaftlich in hohem Maße von Relevanz ist und etwa von Arend Lijphart (1968) als Geheimrezept für die (Über-)Lebensfähigkeit liberaler Demokratien in tief gespaltenen Gesellschaften angesehen wird.

Vor dem Hintergrund des zivilgesellschaftlich fundierten Hegemoniekonzepts entfällt bei Gramsci zudem die Staatsfixierung Marxscher Provenienz: Als Mittel zur Erlangung gesellschaftlicher Hegemonie ist die Ideologie umstritten, prekär und deswegen politisch. Gramsci spricht nicht aus, was Laclau und Mouffe später sagen werden: dieser Ideologiebegriff hängt nicht mehr am Konzept der Klasse; er ist aufgegangen in den sozialen Praktiken der Akteure, die um Zustimmung kämpfen.

Kurz gesagt, dort steckt die Ideologie auch nach Auffassung von Althusser. Und ähnlich wie Gramsci will auch Althusser zur Frage nach den Grundlagen der Klassenherrschaft vordringen: Zweifellos, aus der Logik des Marktes speist sich nach marxistischer Auffassung die bürgerliche Gesellschaft, die sich durch staatliche Zwangsgewalt absichert. Aber lässt sich dadurch auch die Reproduktion der Produktionsbedingungen gewährleisten? Nein, so Althusser (1977: 122) in Anlehnung 
an Gramsci: Repression reicht dazu nicht aus, vielmehr bedürfe es zusätzlich der Ideologie - aber diese Aufgabe vollzieht sich nicht mehr als zäher „Stellungskrieg“, sondern unterhalb jeder Aufmerksamkeit zivilgesellschaftlicher Öffentlichkeit (Lloyd 2003: 227). Die ideologische Reproduktionsmaschinerie ist eingelassen in die staatlichen Strukturen. Dort dient sie der Ausbeutung der Arbeiterschaft; hierin folgt Althusser Marx. Nur sie ist seines Erachtens keine bloße Illusion der Realität, die immer auch einer „Allusion“ bedürfte (34). Vielmehr bezeichnet Ideologie immer schon den ,imaginären Charakter" (134) der Repräsentation, und zwar sowohl im Selbstverhältnis als auch im Verhältnis zur Welt. Nur warte „dahinter“ keine Realität, sondern ein „Nichts“, das in der Repräsentation zur Differenz wird und eine (imaginäre) Identifikation ermöglicht. Der Einzelne ist auf Bilder angewiesen - und daher immer schon in besonderer Weise ansprechbar für eine ideologische Bestimmung (Eagleton 1993: 167). Der Status der Ideologie ist daher Althusser zufolge viel autonomer (weil selbstbezüglicher) zu denken, als es das Basis-Überbau-Modell suggerieren mag. Ihre transformative Kraft schafft ihrerseits Realitäten und setzt konsequenterweise selbst eine „materielle Existenz“ voraus (Althusser 1977: 136), die in den „Apparaten“ des Staates und der vielfältigen Formen der Herrschaftspraxis wirkt, und zwar vornehmlich als Modus der Routine, Gewohnheit und Wiederholung. In diesen Erscheinungsweisen der Selbstverständlichkeit wird der Einzelne zum Subjekt geformt, indem er als solches angesprochen wird (,Interpellation“) - das weiß, was es zu tun hat: „Knie nieder, bewege die Lippen zum Gebet und Du wirst glauben“, zitiert Althusser Pascal (138). Die Autonomie des Subjekts als Erfindung des autonomen Betriebs der Ideologie.

Zieht man eine kurze Summe aus den bisherigen Positionen, so sticht zunächst die produktive, auch konstitutive Bedeutung ins Auge, die der Ideologie zugeschrieben wird, und zwar nicht nur in der Gestaltung sozialer und politischer Verhältnisse, sondern ebenso in der Sicherung der eigenen Einflussbedingungen. Im engen Zusammenhang damit steht überdies die Pluralität der Erscheinungsformen, die bei Mannheim und Gramsci auf den gesellschaftlichen Bereich bezogen sind, bei Althusser hingegen auf den Staatsapparat. Mit dieser Unterscheidung geht auch eine grundsätzliche Differenz in funktionaler Betrachtung einher. Die Binnendifferenzierung des staatlichen Ideologiebetriebes führt bei Althusser - ungeachtet seiner konzeptionellen Innovationen und Reformen gegenüber einer klassisch-marxistischen Position - nicht zu einer funktionalen Pluralisierung, während sie bei Mannheim zum Bestandteil der gesellschaftlichen Ausdifferenzierung wird. Vielleicht trägt es zur Popularität von Gramscis Hegemoniekonzept bei, dass er in dieser Frage eine Mittelstellung einnimmt: deskriptiv und doch kritisch. 


\section{Ideologie und Politische Theorie}

Die Ideologieforschung hat sich in den letzten zwei Jahrzehnten zu einem multidisziplinären Forschungsfeld entwickelt, das seit 1996 mit dem Journal of Political Ideologies zudem über eine eigene Fachzeitschrift verfügt. Multidisziplinarität heißt nicht Inter- oder gar Transdisziplinarität; und tatsächlich wird man als bestimmende Struktur dieses Feldes vor allem den (auch anderswo anzutreffenden) Gegensatz zwischen „Qualis“ und „Quantis“ ansehen, der zudem durch die Bipolarität des Ideologiebegriffs (deskriptiv-analytisch vs. pejorativ-kritisch) in der im engeren Sinne politiktheoretischen Diskussion für noch größere Schwierigkeiten beim Import und Export theoretischer Versatzstücke sorgt (Maynard 2013). Vereinfacht gesagt: Während sich die „Theoretiker" bei - vor allem aus der Parteien- und Wahlforschung bekannten - Links-Rechts-Skalierungen schütteln, die in ihren Augen vom Begriff der Ideologie kaum etwas anderes als eine (eindimensionale) Policy(Selbst-)Zuschreibung übrig lässt, können Die-Hard-Empiristen und Big-DataKomparatisten mit der Komplexität der Ideologie nach Zurichtung durch die Politische Theorie (selbst wenn sie wollten) nur wenig anfangen.

Für den Bereich der Politischen Theorie zeigen jedoch die genannten Positionen - von Marx über Mannheim und Gramsci bis zu Althusser - die Vielfältigkeit und Variationsbreite des theoretischen Zugangs, der nicht notwendig sogleich von der Dichotomie zwischen Beschreibung und Kritik ,geschluckt“ werden muss. Das zeigt sich unter anderem an den gegenwärtig bestimmenden Strängen des Ideologiediskurses im Rahmen der Politischen Theorie. Hierzu zählen vor allem die Begriffs- und Diskursanalysen. Mit Einschränkung ist darüber hinaus auch der genealogische Ansatz von Michel Foucault zu nennen: Foucault hat dem Ideologiebegriff zwar explizit seine Theorietauglichkeit abgesprochen (1978: 34), gleichzeitig jedoch die Fruchtbarkeit des Ansatzes von Althusser durch eigene Studien unter Beweis gestellt (Charim 2002). Das gilt in besonderem Maße für seine Studien zur „Disziplinarmacht“, die den Prozess der zwangslosen Formung des Subjekts über den subtilen Zugriff auf den ,gelehrigen Körper“ als nachhaltige Zurichtung und Dressurleistung zutage fördern (Foucault 1976: 173 ff.; 1978: 104).

Ebenfalls nur indirekter Art sind die Bezüge der Begriffsgeschichte, die sich vor allem mit den Studien von Reinhart Koselleck (u. a. 1979) verbinden. Gleichwohl wird hier das Grundanliegen deutlich, dass diese Forschungsrichtung mit jenen Arbeiten von Michael Freeden (v. a. 1996) und der Cambridge School teilt, in diesem Kontext vor allem von Quentin Skinner (z. B. 1978) vertreten: Im Vordergrund steht die Studie der Entwicklung des politischen Denkens, das seinen Ausdruck in einer spezifischen Begrifflichkeit findet, deren historische Verläufe - Brüche und Ab- 
brüche inklusive - nachgezeichnet werden. Während Koselleck dafür jedoch nicht auf den Ideologiebegriff zurückgreift, spielt dieser bei Skinner als Rahmen der Rekonstruktion des intellektuellen Umfeldes politischer Konventionen und Sprache eine explizite Rolle. Ideologie drückt sich aus in „essentially contested concepts“ (Gallie) und ist daher unmittelbar mit dem Ringen um Deutungsmacht verbunden. Ihrer Funktion nach zielt sie auf (politisch erfolgreiche) Strategien zur Legitimitätsbeschaffung zwecks Durchsetzung eines bestimmten Vokabulars: "[I]t is [...] essentially by manipulating this set of terms [political vocabulary - OWL] that any society succeeds in establishing and altering its moral identity" (Skinner 1974: 294). Ähnlich wie Skinner betont auch Freeden das politische Moment, das der Ideologie inhärent ist und aus dem Kampf um Geltung erwächst. Bei ihm liegt der Akzent jedoch etwas stärker (als etwa bei Skinner) auf der Funktion der „decontestation“, der systematischen Überblendung kontingenter Geltungsansprüche. Um dieser spezifischen Leistung der Ideologie auf die Spur zu kommen, nimmt Freeden nicht nur die politisch bedeutsamen kulturellen Kontexte in den Blick, sondern auch die strukturellen Konfigurationen der jeweiligen Ideologien. Eine zentrale Einsicht dieses „,morphologischen Ansatzes“ besteht darin (Freeden 1996: 20), Ideologien als Verknüpfungen unterschiedlicher begrifflicher Elemente zu betrachten, die sich typischerweise nicht gegenüber anderen Konfigurationen abschotten, sondern über bestimmte Elemente mit diesen in Verbindung stehen. Die daraus resultierende Unschärfe ermöglicht in praktischer Hinsicht ein für die politische Sprache charakteristisches Changieren und Manövrieren im Kampf um Zustimmung und Unterstützung oder im Ringen um (gesichtswahrende) Kompromisse. In theoretischer und methodischer Perspektive besteht die Herausforderung darin, den Kern der jeweiligen Ideologien im fluiden Spiel der stets möglichen Verschiebungen, Positionierungen und Akzentuierungen zu extrahieren.

Dieser Fokus auf die ideologischen Inhalte, der zweifellos in der Tradition der Studien von Mannheim steht, zeichnet Diskusanalysen gerade nicht aus. Ihnen geht es in kritischer Absicht um die kommunikativen Praktiken, mit denen bestimmte Ideologien konstituiert und avanciert werden. Die Übergänge zwischen den einzelnen Varianten (wie auch gegenüber den Begriffsanalysen) sind fließend. Ein gewisses Unterscheidungspotenzial ermöglicht der Blick auf die Methoden: Während die Beiträge der „Critical Discourse Analysis" ${ }^{1}$ typischerweise mit einem linguistischen Instrumentarium arbeiten, stehen poststrukturalistische Ansätze stärker in der Tradition marxistischer Ideologiekritik und nutzen eher Analysetechniken, die

1 Zu dieser Richtung gehören unter anderem Norman Fairclough, Theo van Leeuwen, Ruth Wodak und Teun van Dijk. Als Überblick siehe Wodak/Meyer 2009. 
aus den Literatur- und Kulturwissenschaften stammen. ${ }^{2}$ Ungeachtet der unterschiedlichen Konzeptionen erscheinen Ideologien in beiden Fällen als fester Bestandteil der gesellschaftsbildenden Diskursmacht; sie entspringen einer hybriden Geltungsgenese, die bestimmend für ihre inhärente Ambivalenz ist: zugleich alternativlos und fragwürdig zu sein. Aus diskursanalytischer Perspektive verlangt diese Ambivalenz selbst nach einem ambivalenten Umgang, in dem sie machtheoretisch akzeptiert und sozialphilosophisch kritisiert wird. Im Kontext der poststrukturalistischen Studien sind es vor allem die Arbeiten von Laclau und Mouffe, die explizit eine Verbindung zu Gramscis Hegemonieansatz herstellen, in dem diese Ambivalenz bereits angelegt sei, nämlich in der Entkopplung zwischen Ideologie und Klasse (Laclau/Mouffe 1985). Diese Entkopplung weist den Weg über den Marxismus hinaus, dessen Essentialisierung des Klassenbegriffs ein für die Ziele des Sozialismus genuin politisches Verständnis von Gesellschaft verhindere und damit letztlich die erforderliche gesamtgesellschaftliche Demokratisierung behindere. Wer keinen Blick hat für die veränderten Realitäten und neuen radikaldemokratischen Potenziale (weil er an der historischen, aber überkommenen Führungsrolle der Arbeiterschaft festhalte), versäumt die Möglichkeit, Gesellschaft politisch zu gestalten, so das Credo von Laclau und Mouffe.

\section{Demokratietheorie und Ideologiekritik}

Es ist kaum zu übersehen, dass sich diese Ideologiekritik gegenüber dem „Erfinder“ der Ideologiekritik mit dem Einverständnis paart, dass zur Politik des „Stellungskrieges" zugkräftige Ideologismen notwendig sind. Wasch mir den Pelz, aber mach mich nicht nass? Das Problem, woher die rechtfertigungsfähigen Kriterien der Ideologiekritik stammen sollen, wenn Politik notwendig Diskurs und Diskurs notwendig Ideologie ist, hat Laclau selbst gesehen. Seine Antwort läuft auf einen doppelten Ideologiebegriff hinaus (Laclau 1983): Ideologie als Mehrheitsbeschaffer (demokratisch) und Ideologie als Diskursschließung (undemokratisch). Die Leistungsfähigkeit im Bereich der Politik dürfte unterhalb der Systemdifferenz nur mühsam aufrecht zu erhalten sein. Hingegen als Selbstbefragung des politischen Denkens, sofern es einen theoretischen oder philosophischen Anspruch erhebt (Bohmann/ Gertenbach/Laux 2010), ließe sich dieser Sichtweise eine demokratietheoretische Fundierung der Ideologiekritik abgewinnen. Sie wäre allererst verbunden mit der Anfrage an den Ort der politischen Macht innerhalb der jeweiligen Konzeption, und

2 Siehe hierzu exemplarisch die Beiträge in dem Band von Žižek (2012). Als einflussreiche Vertreter lassen sich darüber hinaus und pars pro toto nennen: Ernesto Laclau, Chantal Mouffe, Yannis Stavrakakis, David Howarth und Aletta Norval. 
zwar durchaus auch im Sinne ihrer „Entgiftung“ (Prinz 2012: 102), wie es Raymond Geuss unter anderem in seiner Auseinandersetzung mit dem „mainstream liberalism“ fordert (Geuss 2008). Eine demokratietheoretische Anfrage könnte auch das Projekt der Wiederaufnahme der Ideologiekritik im Geiste Adornos stärken, das unlängst von Jaeggi (2009) in Angriff genommen worden ist: Denn es liegt nahe, dass die strukturelle Subversion der Verwirklichung von jenen Ideen, die sich im politischen Prozess als anerkennungswürdig herauskristallisieren, gut von der etablierten Trennung zwischen epistemischer und normativer Ebene lebt und daher üblicherweise von Seiten der Theorie keine Nachfragen zur Wirkung und Wirklichkeit der eigenen Standards zu befürchten hat. Eine ideologiekritische Selbstbeschäftigung der Politischen Theorie setzt zweifellos zunächst das Selbstverständnis der Ideologieanfälligkeit voraus - und diese wiederum einen gehaltvollen Ideologiebegriff, der etwa im konservativen Denken alles andere als ausgereift ist (Müller 2006). In dieser Hinsicht bleibt noch so manches Feld zu beackern - aber sich davor „verschließen“" zu wollen, wäre bereits ein Hinweis für einen Anfang.

\section{Literatur}

Aron, Raymond, 1955: Fin de l'àge idéoIogique?, in: Theodor W. Adorno/Walter Dirks (Hrsg.), Sociologica 1. Aufsätze, Max Horkheimer zum sechzigsten Geburtstag gewidmet, Frankfurt a. M., 219-233.

Althusser, Louis, 1977: Ideologie und ideologische Staatsapparate. Aufsätze zur marxistischen Theorie, Hamburg/Berlin.

Barrett, Michèle, 1991: The Politics of Truth: From Marx to Foucault, Cambridge. Bell, Daniel, 1962: The End of Ideology: On the Exhaustion of Political Ideas in the Fifties, New York.

Bohlender, Matthias, 2010: Die Herrschaft der Gedanken. Über Funktionsweise, Effekt und die Produktionsbedingungen von Ideologie, in: Harald Bluhm (Hrsg.), Karl Marx/Friedrich Engels: Die Deutsche Ideologie, Klassiker auslegen, Bd. 36, Berlin, 41-57.

Bohmann, Ulf/Gertenbach, Lars/Laux, Henning, 2010: Ein Spiel zwischen Nähe und Distanz. Formen der Kritik unter nachmetaphysischen Bedingungen, in: Karin Becker u. a. (Hrsg.), Grenzverschiebungen des Kapitalismus, Frankfurt a. M./New York, 55-74.

Bracher, Karl-Dietrich, 1982: Zeit der Ideologien. Eine Geschichte des politischen Denkens im 20. Jahrhundert, Darmstadt.

Charim, Isolde, 2002: Der Althusser-Effekt. Entwurf einer Ideologietheorie, Wien. Crouch, Colin, 2004: Post-Democracy, Cambridge/Malden (MA). 
Eagleton, Terry, 1993: Ideologie. Eine Einführung, Stuttgart/Weimar.

Foucault, Michel, 1976: Überwachen und Strafen. Die Geburt des Gefängnisses, Frankfurt a. M.

Foucault, Michel, 1978: Dispositive der Macht. Michel Foucault über Sexualität, Wissen und Wahrheit, Berlin.

Freeden, Michael, 1996: Ideologies and Political Theory, Oxford.

Fukuyama, Francis, 1992: The End of History and the Last Man, New York.

Geuss, Raymond, 2008: Philosophy and Real Politics, Princeton/Oxford.

Gramsci, Antonio, 1998: Gefängnishefte, hrsg. von Klaus Bochmann und Wolfgang

Fritz Haug/Peter Jehle, Band 8, Heft 19 (1934/35), Hamburg.

Jaeggi, Rahel, 2009: Was ist Ideologiekritik?, in: Rahel Jaeggi/Tilo Wesche (Hrsg.),

Was ist Kritik? Frankfurt a. M., 266-295.

Kennedy, Emmet, 1978: Destutt de Tracy and the Origins of "Ideology", Philadelphia.

Kojève, Alexandre, 2005: Hegel. Eine Vergegenwärtigung seines Denkens. Kommentar zur „Phänomenologie des Geistes“, erweiterte Ausgabe, Frankfurt a. M.

Koselleck, Reinhart, 1979: Vergangene Zukunft. Zur Semantik geschichtlicher Zeiten, Frankfurt a. M.

Laclau, Ernesto, 1983: The Impossibility of Society, in: Canadian Journal of Political and Social Theory 7 (1-2), 21-24.

Laclau, Ernesto/Mouffe, Chantal, 1985: Hegemony and Socialist Strategy. Towards a Radical Democratic Politics, London.

Lijphart, Arend, 1968: The Politics of Accommodation: Pluralism and Democracy in the Netherlands, Berkeley (CA).

Lipset, Seymour Martin, 1990: Consensus and Conflict. Essays in Political Sociology, 2. Aufl., New Brunswick (NJ).

Lloyd, Moya, 2003: The End of ideology?, in: Robert Eccleshall et al. (Eds.), Political Ideologies: An Introduction, 3. Aufl., London/New York, 217-241.

Mannheim, Karl, 1995: Ideologie und Utopie, 8. Aufl., Frankfurt a. M. [erstmals Bonn 1929].

Marx, Karl/Engels, Friedrich, 1958: Die deutsche Ideologie (1845/46), in: MarxEngels Werke (MEW), Bd. 3, Berlin.

Maynard, Jonathan Leader, 2013: A map of the field of ideological analysis, in: Journal of Political Ideologies 18 (3), 299-327.

Müller, Jan-Werner, 2006: Comprehending Conservatism: A New Framework for Analysis, in: Journal of Political Ideologies 11 (3), 359-365. 
Nietzsche, Friedrich, 1955: Also sprach Zarathustra (1883-1885), in: Karl Schlechta (Hrsg.), Werke in drei Bänden. Bd. 2: Die fröhliche Wissenschaft. Also sprach Zarathustra u. a., München.

Prinz, Janosch, 2012: Zwischen kritischer Reflexion und Handlungsorientierung. Gespräch mit Raymond Geuss zu seinem Ansatz in der politischen Philosophie, in: Zeitschrift für Politische Theorie 3 (1), 95-108.

Ritzi, Claudia, 2014: Die Postdemokratisierung Politischer Öffentlichkeit: Kritik Zeitgenössischer Demokratie - Theoretische Grundlagen und Analytische Perspektiven, Wiesbaden.

Schirrmacher, Frank, 2011: „Ich beginne zu glauben, dass die Linke recht hat", Frankfurter Allgemeine Sonntagszeitung, 15. August 2011, http://www.faz.net/ aktuell/feuilleton/buergerliche-werte-ich-beginne-zu-glauben-dass-die-linkerecht-hat-11106162.html (Stand: 4.9.2014).

Shils, Edward, 1955: The End of Ideology?, in: Encounter 5 (November), 52-58.

Skinner, Quentin, 1974: Some Problems in the Analysis of Political Thought and Action, in: Political Theory 2 (3), 277-303.

Skinner, Quentin, 1978: Foundations of Modern Political Thought. Bd. 1: The Renaissance, Cambridge.

Wodak, Ruth/Meyer, Michael, 2009: Critical Discourse Analysis: History, Agenda, Theory and Methodology, in: Dies. (Hrsg.), Methods of Critical Discourse Analysis, 2. Aufl., London, 1-33.

Wolin, Sheldon, 1996: Fugitive Democracy, in: Seyla Benhabib (Hrsg.), Democracy and Difference, Princeton, 31-45.

Žižek, Slavoj (Hrsg.), 2012: Mapping Ideology, London/New York.

Korrespondenzanschrift:

Dr. Oliver W. Lembcke

Friedrich-Schiller-Universität Jena

Institut für Politikwissenschaft

Carl-Zeiss-Str. 3

07737 Jena

E-Mail: oliver.lembcke@uni-jena.de 